\title{
Group Theory, Probability, the Structure of Spacetime and V. S. Varadarajan
}

\author{
I. V. Volovich* \\ Steklov Mathematical Institute, Gubkina 8, 119991 Moscow, Russia \\ Received December 29, 2015
}

DOI: $10.1134 / \mathrm{S} 2070046616010015$

This issue of the journal and the previous one (Volume 7, Issue 4, 2015) contains articles presented at the conference "Group Theory, Probability, and the Structure of Spacetime" held at the University of California at Los Angeles, November 7-9, 2014, on the occasion of the retirement of Prof. V. $\mathrm{S}$. Varadarajan. The authors are the world-class researchers in the fields of representation theory of Lie and super Lie algebras and groups, supersymmetry and supersymmetric theories, as well as in representations of Lie groups over non-Archimedean fields and their applications to structure of spacetime and the study of dynamical processes in spacetime. Prof. V. S. Varadarajan has made an outstanding contribution to these fields over the past fifty years.

${ }^{*}$ E-mail: volovich@mi.ras.ru 